Revue interdisciplinaire sur la Grèce archaïque

24 | 2021

L'antropologo "classico» entre bêtes et dieux. Omaggi a Ezio Pellizer (Vol. 1)

\title{
«Qui me délivrera des Grecs et des Romains ?»
}

"Qui me délivrera des Grecs et des Romains?"

"Qui me délivrera des Grecs et des Romains?"

\section{Maurizio Bettini}

\section{OpenEdition}

\section{Journals}

Edizione digitale

URL: https://journals.openedition.org/gaia/1743

DOI: $10.4000 /$ gaia. 1743

ISSN: 2275-4776

\section{Editore}

UGA Éditions/Université Grenoble Alpes

\section{Edizione cartacea}

ISBN: 978-2-37747-292-5

ISSN: $1287-3349$

Notizia bibliografica digitale

Maurizio Bettini, ««Qui me délivrera des Grecs et des Romains ?»», Gaia [Online], 24 | 2021, online dal

31 mai 2021, consultato il 09 décembre 2021. URL: http://journals.openedition.org/gaia/1743 ; DOI: https://doi.org/10.4000/gaia.1743

Questo documento è stato generato automaticamente il 9 décembre 2021.

Gaia. Revue interdisciplinaire sur la Grèce archaïque 


\title{
«Qui me délivrera des Grecs et des Romains ?»
}

\author{
"Qui me délivrera des Grecs et des Romains?" \\ "Qui me délivrera des Grecs et des Romains?"
}

\section{Maurizio Bettini}

Questo elegante alessandrino è stato citato centinaia di volte nel corso della secolare polemica fra chi voleva farla finita, una buona volta, con i classici, e chi invece si ostinava a difenderne l'importanza. Si tratta di un verso talmente fortunato da essersi guadagnato una menzione nei repertori di detti celebri, dunque è facile scoprire che a scriverlo fu Joseph de Berchoux, un letterato e umorista francese nato il 3 novembre 1760 nella Maison de la Forest à Saint-Symphorien-de-Lay (Loire) e morto il 17 dicembre 1838 a Marcigny (Saône-et-Loire). Durante la sua vita Berchoux ha dunque attraversato la tempesta della Rivoluzione, ha assistito ai fasti di Bonaparte e alla sua rovinosa caduta, ha conosciuto la Restaurazione... Non c'è che dire, colui che invocò la liberazione dai Greci e dai Romani ha condiviso uno dei periodi più convulsi della storia europea. Ma se, come dicevo, è facile scoprire chi è l'autore di quel celebre verso, meno facile è leggere per intero il contesto di cui esso fa parte, anche perché difficilmente qualcuno si preoccupa di riportarlo. Andando a curiosare nel quarto volume del Censeur théatrâle, apparso a Parigi nell'anno 1798, ho dunque potuto accertare che «Qui me delivrera des Grecs et des Romains» costituisce l'incipit di un poemetto in alessandrini, con rime rigorosamente baciate, presentato dal curatore sotto la dicitura Élégie sur les anciens et les modernes, écrite par un auteur du dix-huitième siècle ${ }^{1}$. La stessa opera compare però in una raccolta di poesie, stavolta esplicitamente presentata sotto il nome di Joseph Berchoux, apparsa a Parigi nel $1805^{2}$. Ecco anzi alcune parti di questo poemetto nella traduzione italiana che ne abbiamo curata per dedicarla a Ezio, ricordando il suo amore per l'ironia, prima di tutto, e per la letteratura francese:

Chi mi libererà dai Greci e dai Romani?

Che dalle loro tombe, oh, popoli inumani,

di sicuro faranno della mia vita il male:

ascoltate vi prego, queste tristi parole.

Appena ch'io fui nato, il latin rudimento 
suppliziò la mia infanzia con crudo accanimento.

Fu la lingua dei Cesari la mia torturatrice,

di quanto preferivo quella della nutrice!

Per più di dieci anni son stato messo in croce

e la causa fur Livio, Cicerone e Nepote,

quei Romani sepolti da quattrocento lustri

che io maledicevo, coi lor libri vetusti.

[...]

Certo è stato ben peggio quando dentro ai teatri

io non sentivo d'altri che di Fedra e Cleopatra,

d'Arianna e Didone, dei loro amanti e sposi,

tutti quanti arrabbiati come dei lupi, irosi,

Rodoguna, Giocasta, e tutti i Pelopídi

e poi tanti altri eroi, nobili parricidi.

[...]

E tu, trista famiglia, fonte d'eterni lai,

stirpe d'Agamennòn, che non finisce mai,

di voi io udivo ovunque omicidi e assassini,

sanguinarie violenze - in versi alessandrini.

[...]

Dei miei contemporanei mi volevo occupare,

ma gli antichi non mi hanno lasciato respirare.

Se dunque Berchoux si sentì a tal punto soffocato dai Greci e dai Romani, la colpa fu essenzialmente della grammatica latina subita durante l'infanzia (il 'rudimento' delle sue regole) e della sovrabbondanza di personaggi e trame che il teatro a lui contemporaneo traeva dalle vicende dell'antichità: troppe Giocaste, troppe Fedre, troppe Cleopatre. Diciamo subito però che almeno sul primo punto, ossia l'antipatia per la grammatica latina, Berchoux è in buona compagnia. Basta ricordare cosa scriverà Heinrich Heine, alcuni decenni dopo di lui, rievocando il periodo in cui da bambino studiava il latino a scuola. Il piccolo Heine infatti non riusciva a tenere a mente $\mathrm{i}$ sostantivi irregolari della terza declinazione. Quelli che, essendo per l'appunto 'irregolari', hanno l'accusativo in -im e l'ablativo in -i: tussis, amussis, vis, buris, e via di seguito. Per cui, passando tutte le mattine di fronte a un Cristo crocifisso di legno grigio («una grande immagine squallida che ancor oggi, certe notti, passa nei miei sogni e mi guarda triste con occhi fissi e sanguinanti»), il piccolo Heine implorava: «O tu, povero Dio ugualmente torturato, vedi se ti è possibile che io tenga a mente i sostantivi irregolari!»3. In verità la lista dei disgustati dalla grammatica latina sarebbe lunga, soprattutto se potessimo tener conto di tutti coloro che non hanno mai messo per iscritto le proprie frustrazioni e si sono limitati a confessarle, ormai adulti, ad amici, parenti, coniugi o amanti. Questa lista però, se a qualcuno venisse mai in mente di redigerla per davvero, dovrebbe comprendere almeno Samuel Butler, lo scrittore vittoriano che andò spesso controcorrente rispetto all'epoca sua. Anche nel caso di Butler, infatti, l'odio per il latino fu dovuto alle torture grammaticali subite durante l'infanzia. Nella finzione del suo romanzo più celebre, Erewhon, il latino assume il nome di 'hypotetics', la lingua vana ed assurda il cui apprendimento era imposto ai giovani nel mitico e remoto stato di Erewhon, appunto. Un paese che per la verità tanto somigliava all'Inghilterra del tempo suo ${ }^{4}$. I lamenti di Berchoux, di Heine o di Butler dovrebbero far risuonare almeno un campanello nella testa di chi si ostina a pensare che insegnare il latino, nella nostra scuola, significhi semplicemente impartirne i 'rudimenti' grammaticali ad allievi distratti. Cosa che come abbiamo visto provoca solo 
il desiderio di sbarazzarsi al più presto dei Greci e dei Romani; ovvero suscita la convinzione che Gesù sia stato inchiodato alla croce a suon di sostantivi irregolari.

3 Tornando a Berchoux, c'è da dire che la sua ostilità verso i Greci e i Romani doveva avere anche ragioni politiche. Verso la fine della sua Élégie, infatti, il poeta lamenta che i moderni, seguendo l'esempio degli antichi, avevano riempito le piazze di beceri Demosteni, di infami Bruti, di Ciceroni grossolani, i quali aizzavano la ferocia delle folle facendo dell'assassinio una virtù; mentre la rapina veniva giustificata in base al fatto che a Lacedemone tutti i beni erano in comune. Gli orrori della Rivoluzione, insomma, per Berchoux sarebbero stati provocati dai detestati Greci e Romani - «ci han sgozzato i Moderni a suon di frasi Antiche!» recita un altro dei suoi mirabili alessandrini. Berchoux comunque non fu l'unico a individuare nel culto per le virtù politiche dei Greci e dei Romani uno dei motivi che condussero la Rivoluzione ai suoi eccessi. Dopo la caduta di Robespierre e l'avvento del periodo termidoriano, l'interpretazione del Terrore come un prodotto dell'ossessione per l'antichità divenne abbastanza corrente: i rituali, nomi, miti e simboli classici adottati dai rivoluzionari lo manifestavano del resto chiaramente. In una lezione tenuta all'École Normale Superieure da ConstantinFrançois de Chassebœuf, conte di Volney, nel 1795, questo storico, filosofo e orientalista francese (dunque uno studioso, non un brillante umorista come il nostro Berchoux) aveva criticato duramente l'eccesso di educazione classica impartita ai giovani durante tutto il secolo, accusandola di aver infiammato i loro animi con gli esempi offerti dai Greci e dai Romani. Attraverso di essi era sviluppata un'adorazione praticamente superstiziosa nei confronti degli antichi, tale da aver suscitato nei giovani un entusiasmo cieco e foriero di violenza. Come diceva Berchoux, insomma, "ci han sgozzato i Moderni a suon di frasi Antiche!». In Francia, dunque, nei decenni successivi alla fine della Rivoluzione si procedette a una vera e propria revisione del paradigma fin qui adottato nell'insegnamento dei classici - e del latino in particolare - nelle scuole francesi. Non potendosi più invocare l'importanza dello studio dei Greci e dei Romani in quanto modelli di virtù civili e morali - dati gli eccessi a cui questo culto dell'antichità aveva condotto - nel sistema educativo dell'Ottocento ci si orientò piuttosto sul valore 'ginnico' del latino, sulla sua pretesa capacità di sviluppare le capacità mentali dei discenti e sulla particolare importanza, in questa prospettiva, posseduta dalla pratica della versione. In altre parole venne elaborato in Francia il modello del latino inteso come 'lingua logica'. I classici, un dì modello di virtù morali, si trasformavano così in palestra della mente, un modo come un altro per salvare il latino dal naufragio. E pensare che in Italia, ancora oggi, c'è gente che considera questo modo di insegnare le materie classiche nella scuola - i poteri taumaturgici della lingua, della traduzione, anzi della versione - come l'unico possibile, quasi che non ne fosse mai esistito, e non potesse mai esisterne, nessun altro ${ }^{5}$.

Come reagì Berchoux al denunciato strapotere dei classici, alla loro arroganza linguistica, teatrale, alla loro rovinosa influenza sulla pratica rivoluzionaria? In verità si rassegnò presto, fece pace con i Greci e con i Romani - e si dedicò all'arte della cucina. Proprio così, alla cucina. Divenne famoso infatti per la pubblicazione di un poema in quattro canti intitolato Gastronomie ou l'homme des champs à table, in cui esaltava i piaceri del palato, sempre, com'è ovvio, in impeccabili versi alessandrini ${ }^{6}$. Per la verità un'opera dallo stesso titolo, Gastronomía (o anche Hedypátheia) l'aveva già scritta Archestrato di Gela, un poeta greco vissuto nel IV secolo a.C., e si trattava di un poemetto che come quello di Berchoux era dedicato alle delizie del palato. Una 
elegante, e divertente, parodia di Omero, in cui i nomi dei pesci più prelibati ricevono gli epiteti che normalmente toccherebbero ad Ettore o ad Achille. Berchoux dichiara anzi esplicitamente, e anche onestamente, che il suo poema si muoveva nel solco tracciato da Archestrato, 'poeta laureato' al tempo dei Greci ${ }^{7}$. Non sappiamo però se il nostro fosse anche informato del fatto che il pater Ennius, poeta vissuto fra III e II secolo a.C., dunque ancor più morto e sepolto di Cicerone e Nepote, aveva brillantemente riadattato nella lingua di Roma il poemetto di Archestrato con il titolo di Hedyphagetica «Cose buone da mangiare», facendo perfino dell'elegante ironia su se stesso autore degli epici Annales. Del resto, visto che Ennio era stato il primo poeta epico latino in esametri, di cos'altro avrebbe potuto far la parodia se non della sua propria opera? A riprova del fatto che questi classici tanto severi e asfissianti poi non erano. In ogni caso dobbiamo concludere che Berchoux, nonostante tutta la buona volontà che inizialmente ci aveva messo, dai Greci e dai Romani non riuscì mai a liberarsi. L'opera che lo rese celebre nel suo paese altro non era, in definitiva, se non una imitazione di quanto i deprecati Antichi avevano già fatto prima di lui. $\mathrm{E}$ anzi, con una buona dose di faccia tosta, bisogna dire, Berchoux non aveva esitato a costellare i suoi alessandrini gastronomici con altrettanti riferimenti all'antichità, citando i banchetti di Patroclo e di Achille, il porco arrostito che fu divorato da Ulisse, la golosità di Lucullo, quella di Apicio, il rombo a proposito del quale Domiziano consultò addirittura il Senato («con quale salsa lo accompagnereste, patres conscripti?»). Proprio come se non ci fosse stato mai nessun problema con i Greci e i Romani e lui non li avesse mai maledetti. Il fatto è che ai classici non si può sfuggire. Dopo aver snocciolato tanti esempi di ghiottoneria greco-romana, Berchoux sembrerebbe avere un momento di resipiscenza, quando afferma:

[...] ho parlato dei Greci, ho citato i Romani, ma questo più non basta ai miei contemporanei.

Prepariamo la cena per l'età a noi vicina!

I poeti hanno troppo sdegnato la cucina.

5 Attenzione però. Se il poeta dichiara che intende distogliere lo sguardo dall'antichità questo non avviene più perché Greci e Romani sono la causa di torture grammaticali e teatrali, o perché le loro mani grondano del sangue innocente versato dai bravi francesi. Al contrario, il poeta interrompe il dialogo con gli Antichi - che peraltro ha tenuto aperto fino a questo momento - solo perché i contemporanei, gente che digerisce bene, hanno voglia di sentir parlare di buoni piatti e di sapida digestione. Berchoux pare davvero uscito da un racconto di Balzac. Sembra uno di quei provinciali soddisfatti del proprio benessere che si godono un bicchiere di cognac dopo un pasto sempre troppo abbondante:

Che nulla mai disturbi il brav'uomo che pranza.

Bene è rider di tutto, senza offender la panza:

una poesia giammai contò più di una cena.

6 Greci, Romani, antichi, moderni, polemiche grammaticali, letterarie, politiche... Lasciamo perdere, la cosa più importante è mangiar bene.

7 Non c'è dubbio che Joseph Berchoux abbia trovato un modo piuttosto originale di accostarsi al mondo greco e romano. Colui che un dì esecrò Greci e Romani aveva inaugurato la via culinaria all'antichità, se così posso definirla, riscrivendo in chiave gastronomica Omero, la tragedia e la storia di Roma. Non credo però che Berchoux sarebbe da prendere ad esempio da parte di chi oggi volesse proporre nuove vie per l'apprendimento delle lingue classiche nella scuola. Va bene prendere le distanze dalla 
didattica tradizionale, ma non vorrei che nell'insegnamento del latino la grammatica fosse sostituita dalle ricette di Apicio. In un certo senso, dopo aver seguito per intero la sua parabola poetica, avremmo quasi preferito che Berchoux restasse un nemico dei Greci e dei Romani, senza far pace con loro sventolando un berretto da cuoco. Soprattutto oggi, infatti, la sua visione gastronomica della classicità potrebbe risultare pericolosa. In giro c'è già troppa gente che legge il mondo in chiave culinaria. Basta pensare al numero strabocchevole di personaggi che, ogni giorno di più, occupano gli spazi della comunicazione agitando padelle, annusando formaggi biologici e affettando pomodorini dop di Boscotrecasucce. Quello che temo, insomma, è che un giorno in televisione si presenti lo chef Cannavacciuolo a spiegarmi che Dante aveva scelto Virgilio come guida nel suo viaggio oltremondano perché lo ammirava in quanto autore di ricette. Proprio così, ed ecco la prova. Non è forse vero che, giunti finalmente alle foci del Tevere, i Troiani mangiarono le loro mense, come aveva predetto l'arpia Celeno? Ed ecco l'interpretazione autentica di questi versi: in realtà le mensae dei Troiani erano formate da tielle di Laurento (biologiche, a lievitazione naturale, farina del Senatore Cappelli) distese su un letto di erbe fini. È disponibile anche la tiella per celiaci. All'epoca questa ricetta ebbe un tale successo da essere considerata, appunto, una profezia divina. E questo Dante lo sapeva.

8 Pensiamo di aver scherzato abbastanza, anche se (come abbiamo già detto) osiamo sperare che a Ezio questo gioco non sarebbe dispiaciuto. In ogni caso, vorremmo adesso sviluppare un argomento più serio a sostegno della nostra tesi, ossia che non c'è alcun bisogno di essere liberati dai Greci e dai Romani. E anzi, che farlo sarebbe un grave errore. A questo scopo riteniamo però importante, anzi fondamentale, che la cultura classica venga oggi presentata ai giovani secondo un modello, o meglio una visione, alla cui elaborazione gli studi di Ezio Pellizer hanno dato un contributo fondamentale. Ossia l'antichità come altro da noi, l'antichità come diversità - i Greci e i Romani come ce la presenta l'antropologia del mondo antico. Siamo certi che Ezio sarebbe ancora una volta al nostro fianco in questa battaglia.

9 Nelle società contemporanee - sempre più variegate, composite, intrecciate - è infatti fondamentale che i giovani facciano il prima possibile l'esperienza di ciò che è altro rispetto alla cultura in cui sono cresciuti. Soprattutto, però, è necessario che lo facciano in un modo non banale e occasionale, ma riflessivo, intelligente, attraverso gli strumenti della comparazione e della messa in prospettiva del 'noi' rispetto agli 'altri', cioè quelli che non sono noi. Come abbiamo già sostenuto più volte in passato, riteniamo che lo studio dell'antichità classica possa fornire un'occasione ideale per abituare i giovani a dialogare con l'alterità. La civiltà greca e romana presenta infatti molti tratti che la differenziano profondamente dalla nostra, rendendola 'altra' rispetto a noi: un modo diverso di concepire la società (basta pensare alla pratica dello schiavismo), una diversa struttura della famiglia, un ruolo e una posizione assegnati alla donna che (per fortuna) non condividiamo più, un diverso modo di concepire la sessualità e così via. Nello stesso tempo però, questi antichi così 'altri' rispetto a noi costituiscono anche una parte cospicua della nostra identità culturale: per il semplice fatto che per millenni noi europei abbiamo continuato a studiare le loro lingue, a leggere le opere che ci hanno lasciato in eredità, a studiare e imitare i canoni della loro arte e del loro diritto, e così via. In questo modo i Greci e i Romani sono entrati dentro di noi, formano in buona parte il midollo della nostra cultura. Quando si ripete che il latino è una lingua morta, ad esempio, si dice in realtà una sciocchezza, perché questa lingua non solo continua a vivere nell'italiano, nel francese, nel castigliano, nel 
catalano e così via, ossia nelle lingue romanze; ma anche in quelle lingue che, come l'inglese, pur non derivando dallo stesso ceppo hanno poi attinto largamente al lessico di Cicerone, di Seneca e del diritto romano. Marcel Proust, del resto, lo aveva già detto a proposito del meraviglioso francese in cui scriveva la Recherche: «Quelle parole che siamo così fieri di pronunziare con esattezza, non sono forse [...] altrettanti errori fatti da bocche galliche, le quali pronunciavano a rovescio il latino? La nostra lingua non è che la pronuncia difettosa di alcune altre» ${ }^{8}$. La vicinanza al latino delle lingue romanze non è provocata semplicemente dal fatto che esse derivano direttamente dal latino, come sappiamo; ma perché in Europa lo studio ininterrotto di questa lingua, da parte delle classi colte, ha fatto sì che lessico e sintassi continuassero a essere plasmati $\mathrm{e}$ arricchiti da un ininterrotto ritorno verso la propria origine. In altre parole, le lingue romanze non hanno mai smesso di ri-latinizzarsi. Non abbiamo forse continuato a studiare il latino per secoli?' ${ }^{9}$ È raro trovare un manuale di letteratura italiana che non registri il trito aneddoto di Manzoni che andò a risciacquare in Arno i propri panni. Benissimo. Nessun manuale, però, si degna di ricordare le centinaia di scrittori, artisti e scienziati, le migliaia di preti, notai, medici, avvocati, bargelli, podestà, perfino capitani di ventura o dentisti, e chi più ne ha più ne metta, che nei secoli passati e fino a ieri hanno sistematicamente risciacquato il proprio linguaggio nella latine acque del Tevere. Imparando cioè a scrivere e parlare come si deve attraverso le frasi di Cesare o di Cicerone, e dunque ri-latinizzando, ad ogni generazione, la lingua italiana. Tutto ciò provoca delle conseguenze di grande importanza, che vanno ben al di là del far rilevare agli studenti - spesso peraltro con loro lieto stupore - il numero di parole italiane che hanno una trasparente etimologia latina; ovvero la matrice profondamente latina dell'aspetto più astratto della nostra articolazione linguistica, la struttura sintattica dell'italiano (quando ancora ci ricordiamo che esiste, usiamo il congiuntivo proprio come facevano i Romani). Diciamo piuttosto che questa continuità fra l'universo linguistico latino e il nostro, corrisponde anche a una continuità di pensiero, implica uno stesso e condiviso modo di segmentare e rappresentare la realtà attraverso il linguaggio. Conoscere il latino, approfondirne con intelligenza il lessico e la sintassi, significa contemporaneamente esplorare una parte consistente del nostro universo intellettuale. Come quando, per vedere con che faccia ci svegliamo al mattino, ci si mette di fronte a uno specchio.

10 Ma non si tratta solo della lingua, ovviamente. Come dicevamo infatti attraverso lo studio ininterrotto delle opere classiche la civiltà europea ha sviluppato un modo di pensare, di vedere il mondo, di riflettere su se stessa, che è profondamente segnato da quello che Greci e Romani ci hanno lasciato in eredità. Per questo motivo la nostra enciclopedia culturale presenta una fortissima contiguità, anzi continuità, con quella che ci viene dalla civiltà classica. Una continuità che si manifesta attivamente non solo attraverso la lingua, come abbiamo appena detto, ma anche in ragione dei modelli culturali - etici, psicologici, comportamentali, affettivi: ma anche architettonici, artistici, cromatici... - che si sono insediati nella nostra percezione del mondo attraverso l'ininterrotta consuetudine con la classicità. Ora, il fatto che il mondo greco e romano sia contemporaneamente così vicino e così lontano, identico a noi e diverso da noi, a mio parere facilita enormemente questa esperienza dell'alterità che, come dicevo, si fa sempre più urgente per i giovani di oggi. Gli antichi sono degli stranieri ma, contemporaneamente, sono noi - di conseguenza, esperire la loro alterità è molto più facile di quanto potrebbe esserlo se si avesse a che fare con civiltà lontane (soprattutto nel medium del linguaggio) come potrebbero esserlo quella cinese o indiana. Ritengo 
dunque che nella comunicazione dell'antico, a scuola ma anche fuori delle aule scolastiche, sarebbe molto importante, e anche molto interessante, mettere sempre in evidenza questo doppio passo dell'antichità, il suo essere noi e altro da noi, il suo presentarsi come interna ed esterna alla nostra cultura.

11 Fra le caratteristiche che rendono 'altra' la cultura antica rispetto alla nostra, e che dunque meglio si prestano a comunicarne la diversità, non ho menzionato però quella che si presenta forse come la più spiccata: ossia la religione. Per riflettere sull'importanza che ha, o meglio avrebbe, la possibilità di riflettere su questo aspetto della alterità classica, proviamo a formulare un'ipotesi per assurdo. Immaginiamo cioè che in base a un'improvvisa modifica ministeriale in Italia i programmi scolastici prendessero direttamente inizio dal medioevo. Che cosa accadrebbe?

12 I nostri studenti, e dunque i futuri cittadini dello stato italiano, crescerebbero convinti del fatto che la nostra civiltà ha avuto da sempre soltanto una religione, quella cristiana. Ancor peggio, che 'religione' sia semplicemente sinonimo di 'cristianesimo', visto che nella nostra storia, italiana ed europea, a loro conoscenza non ce ne sarebbero mai state altre. Ecco dunque un ulteriore motivo, nient'affatto trascurabile secondo me, per cui non dobbiamo essere liberati dai Greci e dai Romani: perché lo studio dell'antichità classica, delle sue due lingue, delle sue due letterature, delle sue due culture, costituisce l'unica occasione per i nostri studenti di approfondire la conoscenza di una cultura non cristiana. Non sto dicendo questo per una preconcetta posizione laicista, o anti-cristiana, come potrebbe sembrare. Se mai lo sto dicendo per il motivo contrario, ossia perché ritengo che sia fondamentale che i giovani facciano l'esperienza di ciò che è altro, diverso, anche dal punto di vista della religione. In altre parole, sono convinto che soprattutto in una società come quella contemporanea, percorsa da tanti fermenti e conflitti religiosi, non basti esperire l'assenza della religione, ma sia anche necessario conoscere forme di alterità religiosa. Teniamo anzi conto del fatto che nei nostri programmi scolastici non viene concesso alcuno spazio alle scienze sociali. Di conseguenza agli studenti non sono insegnate discipline che, come l'antropologia, potrebbero farli riflettere sul fatto che sulla terra milioni di uomini pensano il divino in maniera differente da cristiani e, soprattutto, su quali implicazioni culturali questa differenza comporta.

Lo studio delle culture antiche costituisce dunque un'occasione irripetibile per sperimentare a scuola l'esistenza di un mondo in cui l'articolazione del divino si fa in un modo in tutto e per tutto differente rispetto al cristianesimo e agli altri due grandi monoteismi. Fra tutti i modi possibili di comunicare oggi la cultura greca e romana, dunque, credo che focalizzare l'attenzione sulla loro facies religiosa sarebbe di straordinaria importanza. Ed è un peccato che a scuola lo si faccia così poco. Si tratterebbe infatti di mettere in luce le caratteristiche di un mondo in cui il divino è plurale, non unico, in cui la divinità non è esclusiva rispetto alle altre divinità, ma anzi viene accettato di buon grado l'ingresso degli dèi altrui nel proprio pantheon, e ci si concede perfino la libertà di tradurre ciascuna delle proprie divinità nella corrispondente divinità di un popolo diverso - Juppiter romano col greco Zeus, Mercurius romano col germanico Wotan, e così via all'infinito. È questo il motivo per cui la classicità non ha mai sperimentato le guerre di religione: Greci e Romani non hanno mai ucciso in nome di Dio o per affermare la supremazia del proprio dio su quello degli altri. Anche il concetto di tolleranza, emerso con così tanta fatica dal sangue di cui l'Europa si è cosparsa durante il conflitto fra cattolici e protestanti, nel 
mondo antico non avrebbe avuto alcun significato, non sarebbe stato neppure compreso: perché dire che si 'tollerano' le religioni o le credenze degli altri, quando si può addirittura farle proprie in ogni momento? È questo lo scenario religioso che sta dietro alle grandi opere classiche che ancora ammiriamo - i poemi di Omero, la tragedia greca, l'Eneide, le Lettere di Seneca. Al contrario, per tornare all'ipotesi per assurdo che formulavamo all'inizio, se lo studio della storia o della letteratura cominciasse direttamente dal medioevo i nostri studenti conoscerebbero solo l'incoronazione di Carlo Magno da parte del Papa, le lotte fra Guelfi (seguaci del papa) e Ghibellini, il Giudizio Universale della Cappella Sistina, l'assolutismo (cristiano) dei sovrani europei e così via; mentre la tradizione letteraria che sta alle nostre spalle finirebbe solo per corrispondere alle maestose invenzioni (cristiane) della Divina Commedia, alle meravigliose malinconie (cristiane) di Torquato Tasso, al cattolicesimo illuminato di Alessandro Manzoni: perfino il pessimismo di Giacomo Leopardi, per essere compreso, ha bisogno di essere proiettato sull'orizzonte cristiano. Ecco perché lo studio e la presenza del mondo antico restano fondamentali nella nostra cultura. Perché ci permettono di sperimentare l'altro, il diverso, anche dal punto di vista della religione. Nell'offerta formativa delle nostre scuole, come viene chiamata, figura una disciplina che porta il nome di 'religione' ed è impartita da insegnanti scelti dalla curia vescovile. Questa disciplina non porta il nome di «religione cattolica», come sarebbe corretto, ma di 'religione' tout court, quasi che quella cattolica fosse in realtà l'unica religione esistente sulla faccia del globo - proprio come se i nostri programmi scolastici prendessero inizio, cronologicamente, dal medioevo. Ebbene, se assieme alla consecutio temporum, all'Eneide di Virgilio e alla tragedia greca si studiasse anche un po' di politeismo antico, facendo riflettere gli studenti sulle caratteristiche peculiari di questa esperienza religiosa, gli studenti potrebbero finalmente inserire la loro 'ora di religione' - che essi la seguano o meno - in un quadro mentale un po' più complesso di quanto solitamente non avvenga. Probabilmente, però, è proprio questo che si vuole evitare.

In realtà ritengo che questa funzione di alterità all'interno della nostra enciclopedia culturale, anche dal punto di vista religioso, il mondo antico l'abbia svolta fin dalla fine della civiltà classica. Si tratta anzi di un fenomeno antropologico su cui varrebbe la pena soffermarsi con più attenzione, per metterne in evidenza almeno il carattere insolito, oltre che l'importanza.

La caratteristica di cui parliamo (seppure in forme diverse, comune anche ad altre culture europee) potremmo infatti descriverla in questo modo: per millenni l'Italia ha fondato e sviluppato la propria cultura su una seconda cultura - quella latina e quella greca - una cultura cioè diversa dalla cultura che più direttamente si esprimeva attraverso le lingue vernacole: una cultura che, anzi, rispetto alla propria è stata a lungo ritenuta superiore. Si tratta di un modello bi-culturale che, in qualche modo, ripeteva quanto era a sua volta avvenuto nella Roma antica, e nei paesi romanizzati, con il ricorso alla lingua e alla cultura greca come seconda cultura, letteraria e filosofica: in grado di alimentare quella 'locale' e, rispetto a essa, ritenuta per molti aspetti superiore. Che la nostra cultura si sia sviluppata così - con un occhio a quanto accadeva 'in casa', un altro rivolto verso l'antichità - lo si può ovviamente riscontrare attraverso numerosi esempi, tratti dalla poesia, dalla filosofia, dall'architettura e così via. Scegliamone uno di argomento religioso, per restare all'interno del tema che ci siamo scelti per svolgere queste ultime riflessioni sulla comunicazione dell'antico oggi. 

definitivamente eliminato. Gli antichi dèi furono relegati al ruolo di vani idoli, se non di demoni, i loro culti prima proibiti poi, inevitabilmente, obliati e cancellati dal tempo. Nel frattempo, però, si verificava un fenomeno singolare. Nel mentre che "gli dèi falsi e bugiardi» venivano rimossi dai loro templi, e questi edifici distrutti o trasformati in chiese cristiane, le testimonianze e i racconti che riguardavano le antiche divinità non venivano affatto distrutti o dimenticati: al contrario. In quanto parte della tradizione letteraria, filosofica e artistica proveniente dall'antichità - e spesso con l'involontaria complicità degli apologeti cristiani - le figure degli dèi 'pagani' e le vicende che li riguardavano hanno continuato non solo a essere tramandate, ma anche a suscitare nuovi racconti e reinterpretazioni. Sia pure moralizzati, allegorizzati, insomma riadattati alle nuove esigenze ideologiche e religiose imposte dal cristianesimo, gli antichi dèi erano rimasti comunque là, non erano mai usciti di scena ${ }^{10}$. Proviamo anzi ad osservare più da vicino che cosa accade allorché Dante, nel primo canto del Paradiso, si trova di fronte alla necessità di cantare ciò che ha visto lassù: «cose che ridire / né sa né può chi di là sù discende».

Il poeta è in difficoltà e non esita a dichiararlo. Il fatto è che contemplando così dappresso il proprio 'desire', cioè Dio, l'intelletto umano «si profonda tanto» che non ce la fa a tener dietro alla memoria. A questo punto, per uscire dalla terribile impasse, non resta che invocare l'aiuto divino, e Dante lo fa, ma in questo modo ${ }^{11}$ :

o buono Appollo, a l'ultimo lavoro

fammi del tuo valor sì fatto vaso,

come dimandi a dar l'amato alloro.

Per essere in grado di 'ridire' ciò che ha visto nell'alto dei cieli, dunque, il poeta non trova di meglio che invocare il dio Apollo. La cosa potrà apparire ovvia solo a chi, per motivi di banalità scolastica, sia abituato a note a piè di pagina del tipo: «Apollo: divinità della mitologia classica. Figura qui come metafora della poesia» ${ }^{12}$. Proviamo invece ad allontanare l'obiettivo. In realtà il comportamento di Dante non è affatto ovvio. Nel poema cristiano per eccellenza, la Divina Commedia - e proprio al momento in cui il poeta si accinge a cantare del massimo 'desire' di ogni intelletto, il Dio cristiano - ecco che a rappresentare la forza ispiratrice della poesia si affaccia la figura di Apollo: una divinità 'pagana', appartenente a una religione severamente bandita dalle coscienze e ufficialmente morta da secoli. Il fatto è che (come abbiamo già detto) gli dèi antichi non se ne erano mai andati, i testi latini che continuavano ad essere letti e studiati garantivano comunque la loro permanenza. In questo modo essi erano pronti a essere rilanciati, con l'avvento dell'Umanesimo e del Rinascimento, nelle nuove avventure artistiche, letterarie e filosofiche che ci sono note sia attraverso il nostro patrimonio culturale 'esterno' (la Nascita di Venere del Botticelli, la Stanza di Amore e Psiche di Giulio Romano) sia attraverso quello 'interno' (le Genealogiae di Boccaccio, la Fabula di Orfeo di Poliziano, le opere di Marsilio Ficino). Non dimentichiamo, però, che tutto questo avveniva nel mentre il cristianesimo e la Chiesa, attraverso i propri Libri Sacri e la propria dottrina, tenevano ben saldamente in pugno le redini della cultura, a ogni livello; e la permeavano di vicende e immagini che erano quelle della Natività o della Passione, quando non erano tratte dal Vecchio Testamento o da leggende di martiri e santi. In altre parole possiamo dire che la nostra cultura ha vissuto per secoli disponendo non di una sola ma di due mitologie, una cristiana, l'altra classica, entrambe attive sul piano artistico, letterario e filosofico; e nei secoli si è continuato a ricorrere a entrambe spesso intrecciandole, altre volte distinguendole - talora polemicamente - 
in questo modo dando comunque vita a una delle manifestazioni più singolari di quella visione binoculare della cultura che è caratteristica della nostra tradizione. Questa vicenda storica così peculiare fa sì che nei nostri Musei i ritratti della Vergine si alternino con quelli di Venere, e anche che sia necessario conoscere la mitologia classica per comprendere ciò che si racconta nel più cristiano dei poemi, la Divina Commedia. Il fatto è che la cultura consegnataci dai secoli passati è a un tempo se stessa (vernacola nei linguaggi, cristiana nella religione) e altra da sé: ossia classica. Quella classica è la nostra seconda cultura. Ecco dunque un altro motivo per tornare a riflettere, con occhi nuovi, su quello che i padri della Chiesa si ostinarono a definire 'paganesimo'.

\section{BIBLIOGRAFIA}

BERCHOUX Joseph, La Gastronomie, poème par J. Berchoux, suivi des poésies fugitives de l'auteur, Quatrième édition, Paris, Giguet et Michaut, 1805a, XIII.

BERCHOUX Joseph, Gastronomie ou l'homme des champs à table, Paris, Guizet et Michaut, 1805b.

BUTLER Samuel, Erewhon, trad. it. di L. Drudi Dembi, Milano, Adelphi, 1975.

CIBOIS Philippe, Latin et politique. Troisième partie : l'impact de la Révolution, in La question du Latin, 4 febbraio 2010. Disponibile su <https://enseignement-latin.hypotheses.org/344>.

DE MAURO Tullio, intervento sul tema Latino perché? Latino per chi?, in «Quaderno dell'Associazione TreeLLLe», 1, 2008, pp. 83-95.

GRIMOD DE LA REYNIÈRE Alexandre Balthazar Laurente (ed.), Le Censeur dramatique, ou, Journal des principaux théatres de Paris et des départemens, par une société de gens-de-lettres, redigé par Grimod de la Reynière, Tome quatrième, Paris, Bureau du Censeur dramatique, 1798.

HEINE Heinrich, Il libro Le Grand, trad. it. di B. Maffi, Milano, Rizzoli, 1956.

PIERONI Silvia, Latino e italiano, in R. Simone (ed.), Enciclopedia dell'Italiano, Roma, Treccani, 2010-2011.

PROUST Marcel, Sodoma e Gomorra, Parte seconda, in Alla ricerca del tempo perduto, trad. it. di N. Ginzburg, Milano, Mondadori, 1970.

SEZNEC Jan, La sopravvivenza degli antichi dèi, trad. it. di P. Gonnelli Miccoli, Milano, BollatiBoringhieri, 1981.

TOMMASEO Niccolò (ed.), Alighieri. La Divina Commedia, III, Torino, UTET, 1944.

VOLNEY Constantin-François de Chassebœuf, conte di, Leçons d'histoire, sixième séance, dites aussi Leçons prononcées à l'École normale en 1795, an III Volney, 1843.

WAQUET Françoise, Latino. L'impero di un segno (XVI-XX secolo), trad. it. di A. Serra, Milano, Feltrinelli, 2004. 


\section{NOTE}

1. Grimod de la Reynière (1798, 182-196).

2. Berchoux (1805a, 165-173).

3. Heine (1956, 6, 134-135).

4. Butler (1975).

5. Volney (1843, 592-593). Vedi Cibois (2010).

6. Berchoux (1805b).

7. Berchoux $(1805 b, 6)$.

8. Proust $(1970,144)$.

9. Cfr. De Mauro (2008, 83-95); Pieroni (2010-2011, 754-759); Waquet (2004).

10. D'obbligo il rimando al celebre studio di J. Seznec (1981).

11. Dante, Paradiso, 1,13 ss.

12. «Apollo e le Muse in Dante sono simboli», notava asciuttamente Niccolò Tommaseo (cito dall'edizione UTET, Tommaseo 1944, 5).

\section{RIASSUNTI}

Il saggio approfondisce la figura di Joseph Berchoux, l'autore del celebre verso «Qui me délivrera des Grecs et des Romains ?». In particolare analizza il contesto storico in cui nacque la polemica da Berchoux contro i classici, ossia la reazione all'uso che degli exempla greci e romani avevano fatto molti rappresentanti della Rivoluzione francese. Nei decenni successivi Berchoux non solo cambiò atteggiamento verso i classici, ma scrisse un fortunato poemetto gastronomico. Il saggio si conclude motivando come, oggi più che mai, i giovani (a dispetto del celebre verso di Berchoux) debbano continuare a studiare i classici come espressione di 'alterità vicina'.

Cet essai explore la figure de Joseph Berchoux, l'auteur du célèbre vers «Qui me délivrera des Grecs et des Romains? » et analyse tout particulièrement le contexte historique dans lequel est née la polémique de Berchoux contre les classiques, c'est-à-dire la réaction à l'usage que de nombreux représentants de la Révolution française avaient fait des exemples grecs et romains. Dans les décennies suivantes, Berchoux a non seulement changé son attitude par rapport aux classiques, mais a également écrit un célèbre poème gastronomique. L'essai se termine en expliquant comment, aujourd'hui plus que jamais, les jeunes (malgré le fameux vers de Berchoux) doivent continuer d'étudier les classiques comme une manière d'expérimenter l'« altérité la plus proche ».

The essay explores the figure of Joseph Berchoux, the author of the famous verse "Qui me délivrera des Grecs et des Romains?". It does so by analyzing the historical context in which Berchoux's polemics against the classics was generated: the reaction to the use of the Greek and Roman exempla by many French Revolutionaries. In the decades which followed, Berchoux not only changed his attitude towards the classics, but even wrote a lucky gastronomic poem. The essay concludes by arguing that, today more than ever, and in spite of Berchoux's famous verse, young people should continue to study the classics as a way to explore the "otherness next door". 
INDICE

Mots-clés : Berchoux, classiques, satire, Révolution française, altérité

Parole chiave : Berchoux, classici, satira, Rivoluzione francese, alterità

Keywords : Berchoux, classics, satire, French Revolution, otherness

\section{AUTORE}

\section{MAURIZIO BETTINI}

Università di Siena, Centro Antropologia e Mondo Antico

maurizio.bettini@unisi.it 